\title{
Iron Succinate Increased Ferritin and Transferrin Saturation in Non-Anaemic Patients with Heart Failure and Iron Deficiency-A Pilot Study
}

\author{
Kurt Boman $^{1,2^{*}}\left(\mathbb{D}\right.$, Mona Olofsson ${ }^{1,2}$ \\ ${ }^{1}$ Research Unit, Department of Medicine, Skellefteå, Sweden \\ ${ }^{2}$ Department of Public Health and Clinical Medicine, Umeå University, Umeå, Sweden \\ Email: *kurt.boman@regionvasterbotten.se
}

How to cite this paper: Boman, K. and Olofsson, M. (2021) Iron Succinate Increased Ferritin and Transferrin Saturation in Non-Anaemic Patients with Heart Failure and Iron Deficiency-A Pilot Study. World Journal of Cardiovascular Diseases, 11, 11-19.

https://doi.org/10.4236/wjcd.2021.111002

Received: November 19, 2020

Accepted: January 10, 2021

Published: January 13, 2021

Copyright $\odot 2021$ by author(s) and Scientific Research Publishing Inc. This work is licensed under the Creative Commons Attribution International License (CC BY 4.0).

http://creativecommons.org/licenses/by/4.0/

(c) (i) Open Access

\begin{abstract}
Introduction: Iron deficiency (ID) is often present (32\% - 65\%) in patients with heart failure (HF). Oral iron absorption in patients with HF is generally poor. This is the reason why oral treatment is not recommended. Aim: To test whether oral iron succinate significantly increases iron deposits in non-anaaemic patients with HF. Methods: ID was defined as ferritin $<100 \mu \mathrm{g} / \mathrm{L}$ or $100-299$ $\mu \mathrm{g} / \mathrm{L}$ with transferrin saturation $(\mathrm{TSAT})<20 \%$. Forty-two patients were screened. Ferrous succinate was administered as one tablet $(100 \mathrm{mg}$, including $32.48 \mathrm{mg} \mathrm{Fe}^{2+}$ plus $100 \mathrm{mg}$ succinate) in the morning and one tablet at bedtime for at least 3 months. Hemoglobin, CRP, ferritin, iron, TSAT, and hepcidin were analyzed before starting treatment, at 6 weeks, and at 3 months end of study (EOS). Results: Five women and 15 men were included in the study. The level of ferritin increased significantly from baseline to 6 weeks (47 to $78 \mu \mathrm{g} / \mathrm{L}, \mathrm{p}=0.009$ ) and baseline to EOS ( 47 to $85 \mu \mathrm{g} / \mathrm{L}, \mathrm{p}=0.001)$. TSAT increased significantly from baseline to 6 weeks $(20 \%$ to $27 \%, \mathrm{p}=0.046)$ and baseline to EOS ( $20 \%$ to $25 \%, \mathrm{p}=0.043$ ). Hepcidin increased significantly from baseline to 6 weeks $(2.5 \mathrm{nmol} / \mathrm{L}$ to $4.8 \mathrm{nmol} / \mathrm{L}, \mathrm{p}=0.006)$ and baseline to EOS ( 2.5 to $4.2 \mathrm{nmol} / \mathrm{L}, \mathrm{p}=0.02)$. Conclusion: Oral iron succinate significantly increased iron uptake, almost doubling the ferritin levels and increasing the TSAT, in patients with HF. Our findings challenge the opinion that oral iron treatment cannot significantly increase iron deposits in non-anemic patients with ID and HF.
\end{abstract}

\section{Keywords}

Iron Deficiency, Non-Anemic, Heart Failure, Iron Succinate 


\section{Introduction}

Iron plays a central role in oxygen availability and usage in the myocardium. In addition to the crucial role of iron in blood formation, it is also important in the Krebs cycle. ID is accompanied by decreased quality of life, functional capacity, and increased morbidity regardless of the presence of anemia [1]. Recent trials of long-term intravenous therapy with ferric carboxymaltose have shown beneficial symptomatic and morbidity effects in patients with iron deficiency (ID) and HF regardless of hemoglobin status [1] [2].

The absorption of oral iron preparations is generally poor in patients with HF and is associated with side effects, which may affect compliance [3]. HF is also regarded as a condition with an increased inflammatory response and subsequent elevation of hepcidin levels, which further impair iron absorption [4]. High dose oral iron polysaccharide was tested in patients with ID and regardless of hemoglobin status and oral iron polysaccharide did not improve exercise capacity over 16 weeks, and had marginal uptake in iron deposits [5]. Their results did not support the use of oral iron supplementation in patients with ID and HF with reduced ejection fraction ( $\mathrm{HFrEF}$ ). The general recommendation as described in the European Society of Cariology guidelines for treatment of ID in patients with $\mathrm{HF}$ is to use intravenous ferric carboxymaltose [6], and oral treatment is not recommended.

For iron absorption, the type of iron complex used is important, as the dissociation of this complex is of great importance to iron uptake. This was shown early by Brise and Hallberg [7]. They found that iron succinate seemed to have the most favorable uptake compared to other iron compounds, possibly through an effect of succinate on intracellular transport mechanisms, regardless of whether iron was given orally or intravenously [8]. New insights into the role of the possible contribution of succinate to blood stem cell function and blood formation indicate the need to study its potential in patients with HF and ID [9]. Using iron succinate may thus challenge the recommendation that oral iron therapy cannot be adequately taken up in patients with HF. As iron succinate has not been tested previously in patients with ID and HF, our hypothesis was that oral iron succinate might give a better iron uptake than in other reported studies of oral iron compounds for the treatment of ID in patients with HF.

\section{Aim}

Our primary aim was to test whether iron succinate could significantly increase iron deposits as indicated by ferritin levels in patients with HF after 3 months of treatment. A second aim was to explore iron uptake after 6 weeks.

\section{Methods}

At the Department of Medicine-Geriatric, Skelleftea, 304 patients (140 hospitalized and 164 outpatients) were registered from 2017 until January 2019 with a primary or secondary diagnosis of HF. Of these, 262 had no ID, or too low he- 
moglobin or other contraindication for participation. Thus, 42 patients was identified from the case records who might be eligible for participation, based on the following inclusion criteria: case record diagnosis of HF based on symptoms, echocardiography (ECHO) verified reduced cardiac function as systolic and/or diastolic dysfunction, ferritin $<100 \mu \mathrm{g} / \mathrm{L}$ or $100-299 \mu \mathrm{g} / \mathrm{L}$ with transferrin saturation $(\mathrm{TSAT})<20 \%$. Both preserved ejection fraction $(\mathrm{EF})$ and reduced $\mathrm{EF}$ were potentially eligible provided there were no contraindications to participate. Those with an $\mathrm{EF} \leq 40 \%$ were classified as $\mathrm{HFrEF}$, and those with $\mathrm{EF}>40 \%$ were classified as HF with preserved $\mathrm{EF}$ (HFpEF). When EF was described as normal or mildly reduced, these patients were classified as having HFpEF. Those with moderately or severely reduced EF were classified as HFrEF. Patients with a primary diagnosis of cancer, dementia, or some other terminal disease were excluded, as were patients planning to undergo intravenous iron treatment or to take part in another study. Patients with anemia (hemoglobin $<120 \mathrm{~g} / \mathrm{L}$ for females and $<130 \mathrm{~g} / \mathrm{L}$ for males) were also excluded because the new indication for treatment of HF is ID regardless of the presence of anemia [1] [2].

The 42 eligible patients were called by phone and informed of the study. After giving oral and written consent to participate, blood sampling was performed to confirm or reject the presence of non-anemic ID, 21 patients did not have ID and one did not want to participate in the study. Thus, 20 patients fulfilled the criteria for enrollment in the study. Patients were managed according to general treatment guidelines at the hospital and/or health care centers. They were instructed not to change their dietary habits.

The study was planned as an open pilot study without placebo control. At the research unit of the Department of Medicine-Geriatric, a special heart failure case record with laboratory data was filled out before starting the study, after 6 weeks, and after 3 months ( \pm 2 weeks; end of study [EOS]). Adverse reactions and side effects were recorded at each visit. Criteria for stopping the treatment were severe side effects or adverse reactions, or the patients wishing to stop the trial. Dosing of ferrous succinate was one tablet $(100 \mathrm{mg}$, including $32.48 \mathrm{mg}$ $\mathrm{Fe}^{2+}$ plus $100 \mathrm{mg}$ succinate) in the morning and one tablet at bedtime for at least 3 months, and not with ordinary meals. Compliance was checked at 6 weeks and 3 month by pill counting.

Blood sampling was performed before 10:00 am according to local and current guidelines. The blood was centrifuged, divided into aliquots, and stored at $-80^{\circ} \mathrm{C}$ in a safe deposit box (Biobank Norr) until analysis. Hemoglobin, C-reactive protein (CRP), ferritin, plasma iron, TSAT, and hepcidin were analyzed before start, at 6 weeks, and at 3 months. Hemoglobin was analyzed locally at each visit. At EOS, hepcidin was analyzed at Laboratory Medicine, Skane, by LC-MS/MS [10] and plasma ferritin, iron, and transferrin were analyzed and TSAT calculated at Laboratory Medicine, Uppsala University Hospital.

From an earlier investigation we had found that patients with HF had a mean ferritin value of approximately $50 \mu \mathrm{g} / \mathrm{L}$ (personell communication). We hypothesized that a clinically meaning increase should be $25 \mu \mathrm{g} / \mathrm{L}$, a relative in- 
crease of $50 \%$. A non-parametric test with $95 \%$ power and a p-value $<0.05$ required at least 16 patients. We assumed that 4 patients may have to stop medication and planned 20 patients for the study. All analyses were performed according to the intention-to-treat analysis. Continuous variables are presented as mean \pm standard deviation for normally distributed values, median and interquartile range for skewed variables, and number and percentage for categorical variables. Due to skewed variables for ferritin and TSAT, a non-parametric test was used for related or independent data. Data were analyzed using IBM SPSS Software version 24 (IBM Corp. Chicago, Illinois, USA).

All patients provided written informed consent, and the ethical committee of Umeå University approved the study (DNR 2018-119-31M). Approval was also obtained from Swedish Medical Product Agency. Diary number 5.1-2018-21157.

\section{Results}

In this study 20 patients participated and the first patient was included on 4 October 2018 and the last on 1 February 2019. The baseline characteristics are given in Table 1.

Table 1. Baseline characteristics of non-anaemic patients with heart failure and iron deficiency.

\begin{tabular}{cc}
\hline Number of patients & 20 \\
\hline Mean age, years \pm SD & $72.7 \pm 8.6$ \\
Male & $15(75)$ \\
Women & $5(25)$ \\
HFpEF $>40 \%$ & $9(45)$ \\
HFrEF $\leq 40 \%$ & $11(55)$ \\
Iron deficiency & $17(85)$ \\
Functional iron deficiency & $3(15)$ \\
History of hypertension & $12(60)$ \\
History of atrial fibrillation & $9(45)$ \\
History of type II diabetes & $9(45)$ \\
History of valvular disorder & $1(5)$ \\
NYHA Class & \\
1 & $1(5)$ \\
2 & $9(48)$ \\
3 & $8(42)$ \\
4 & $1(5)$ \\
Symptoms & $18(90)$ \\
Breathlessness & $16(80)$ \\
Tiredness & $2(10)$ \\
Stomach pain & $4(20)$ \\
Constipation & $1(5)$ \\
Diarrhea & \\
\hline
\end{tabular}

$\mathrm{HFrEF}=$ heart failure with reduced ejection fraction, $\mathrm{HFpEF}=$ heart failure with preserved ejection fraction, NYHA = New York Heart Association. Data are given as n (\%) unless otherwise noted. 
The level of ferritin increased significantly from baseline to 6 weeks (47 to 78 $\mu \mathrm{g} / \mathrm{L}, \mathrm{p}=0.009$ ) and baseline to EOS (47 to $85 \mu \mathrm{g} / \mathrm{L}, \mathrm{p}=0.001$ ). TSAT increased significantly from baseline to 6 weeks $(20 \%$ to $27 \%, \mathrm{p}=0.046)$ and baseline to EOS $(20 \%$ to $25 \%, p=0.043)$. Hepcidin also increased significantly from baseline to 6 weeks $(2.5 \mathrm{nmol} / \mathrm{L}$ to $4.8 \mathrm{nmol} / \mathrm{L}, \mathrm{p}=0.006)$ and baseline to EOS ( 2.5 to $4.2 \mathrm{nmol} / \mathrm{L}, \mathrm{p}=0.026)$. Other laboratory results are given in Table 2 . The patient with the highest ferritin level ( $334 \mu \mathrm{g} / \mathrm{L}$ at 12 weeks, baseline $46 \mu \mathrm{g} / \mathrm{L})$ had an increased hepcidin level at EOS (from 1.1 to $8.8 \mathrm{nmol} / \mathrm{L}$ ). This patient suffered from restless legs, which disappeared during the study without any adverse reactions. Both women and men had significantly increased ferritin levels from baseline to $\operatorname{EOS}(\mathrm{p}=0.043$ and $\mathrm{p}=0.001$, respectively). There were also significant increases in ferritin levels from baseline to EOS in patients with HFpEF and HFrEF ( $p=0.008$ and $p=0.003$, respectively). Four patients stopped the study medication before EOS; three patients because of vomiting or diarrhea and one patient because of hospitalization for a disease other than HF. One patient stopped the study medication after 3.5 weeks but still had an increased ferritin level (from 198 to $237 \mu \mathrm{g} / \mathrm{L}$ ) and TSAT (from $16 \%$ to $21 \%$ ) at EOS. Three patients took only one tablet per day for 2.5, 6, or 7.5 weeks but still had increased ferritin levels from baseline to EOS (38\%, 174\%, and 95\%, respectively). Thirteen patients took the study medication according to the protocol for 12 weeks and had a median ferritin level of $42(32-57.5) \mu \mathrm{g} / \mathrm{L}$ at baseline, $83(61-110.5) \mu \mathrm{g} / \mathrm{L}$ at 6 weeks, and $87 \mu \mathrm{g} / \mathrm{L}(77-139)$ at EOS compared to the median ferritin level of $85 \mu \mathrm{g} / \mathrm{L}$ for the intention-to-treat patients. In those 16 patients who completed the study the study compliance was $>80 \%$.

\section{Discussion}

The main finding was a highly significant increase in ferritin levels after 3 months of iron succinate treatment. TSAT also significantly increased. Thus, the null hypothesis was rejected. Moreover, significant uptake was evident already after 6 weeks. This is in contrast to the recommendation that oral iron substitution cannot improve ID in patients with HF. The increase in ferritin levels seemed

Table 2. Laboratory results for all patients at baseline, six weeks and three months.

\begin{tabular}{cccccc}
\hline & Baseline (IQR) & 6 weeks (IQR) & p-value $^{\mathrm{a}}$ & 3 months (IQR) & p-value \\
\hline Hemoglobin, g/L & $144(135-155)$ & $138(129-150)$ & 0.301 & $142(131-152)$ & 0.461 \\
Iron, $\mu \mathrm{mol} / \mathrm{L}$ & $13.5(10.2-19.8)$ & $16.5(13.2-23.0)$ & 0.102 & $16.0(13.0-24.0)$ & 0.096 \\
Ferritin, $\mu \mathrm{g} / \mathrm{L}$ & $47(32-78)$ & $78(57-113)$ & 0.009 & $85(62-171)$ & 0.001 \\
Transferrin, g/L & $2.6(2.4-2.9)$ & $2.4(2.2-2.7)$ & 0.038 & $2.3(2.0-2.5)$ & 0.009 \\
TSAT, \% & $20(15-30)$ & $27(21-33)$ & 0.046 & $25(21-44)$ & 0.043 \\
Hepcidin, nmol/L & $2.5(0.8-4.4)$ & $4.8(3.0-7.4)$ & 0.006 & $4.2(2.0-8.8)$ & 0.026 \\
hsCRP, mg/L & $2.0(0.9-4.2)$ & $2.0(1.2-5.5)$ & 0.565 & $1.9(0.8-4.1)$ & 0.841 \\
\hline
\end{tabular}

$\mathrm{IQR}=$ interquartile range, $\mathrm{TSAT}=$ transferrin saturation, $\mathrm{hsCRP}=$ high sensitive $\mathrm{C}$-reactive protein. ${ }^{\mathrm{a}}$ Baseline to 6 weeks, ${ }^{\mathrm{b}}$ Baseline to 12 weeks. 
to be of about the same magnitude, regardless of whether the patients suffered from HFpEF or HFrEF. In addition, females and males had similarly increased levels. Our findings challenge the opinion that oral iron treatment cannot increase iron uptake in non-anemic patients with ID and HF.

This is the first study of oral iron succinate in non-anemic patients with both HFpEF or HFrEF and ID, showing significant uptake. Oral iron succinate uptake has not yet been tested in non-anemic patients with HF. A search in PubMed revealed no publications with the search terms iron succinate, iron deficiency, and heart failure. Few studies have compared oral iron therapy and intravenous iron treatment [3]. Another pilot study, the IRON-OUT study [11], tested oral ferrous sulfate (200 mg 3 times daily) vs. intravenous iron sucrose $(200 \mathrm{mg} /$ week for 5 weeks) or placebo in 23 patients with $\mathrm{HF}, \mathrm{EF}<40 \%$, and anemia (9 - 12 $\mathrm{g} / \mathrm{L})$. Ferritin levels increased from $167 \pm 149 \mathrm{ng} / \mathrm{mL}$ to $293 \pm 270 \mathrm{ng} / \mathrm{mL}$ with intravenous iron treatment vs. $115 \pm 141 \mathrm{ng} / \mathrm{mL}$ to $218 \pm 189 \mathrm{ng} / \mathrm{mL}$ in the oral iron group, but the increase in TSAT was less in the oral iron group vs. the intravenous iron group. The main differences from our study were that they tested patients with HF and anemia and high doses of iron sulfate. Another phase 2, double-blind, placebo-controlled randomized clinical trial of patients with HFrEF $(<40 \%)$ and ID compared oral iron polysaccharide $(150 \mathrm{mg}$ twice daily) for 16 weeks and placebo [5]. Ferritin modestly increased by $18 \mathrm{ng} / \mathrm{ml}$ (confidence interval (CI): -8 to 38) with oral iron polysaccharide and $1 \mathrm{ng} / \mathrm{ml}$ (CI: -15 to $17, \mathrm{p}=0.06$ ) with placebo, and TSAT increased $2 \%$ (CI: -3 to 7 ) with oral iron but $0 \%(\mathrm{CI}:-5$ to 5$)$ with placebo $(\mathrm{p}=0.003)$. The main difference from our study was that they included only patients with HFrEF and used iron polysaccharide with a high dose of iron.

The hepcidin levels already increased significantly after 6 weeks and were still significantly elevated at 12 weeks, but with no increase between 6 and 12 weeks. This increase is expected following iron substitution. Gaillard showed that hepcidin increases with both oral iron and intravenous iron, but much more by the intravenous route [12]. Despite this elevation of hepcidin at 6 and 12 weeks, the ferritin level increased from 6 to 12 weeks, though the difference was not significant. As hsCRP did not increase, the reason for the increased hepcidin level should be iron treatment. Hepcidin and CRP levels were in ordinary ranges at baseline, which is probably one reason for the significant uptake of iron succinate in our study. However, one patient with elevated hepcidin and CRP levels also had similar uptake as the patients with ordinary hepcidin and CRP levels. In the study by Lewis [5], patients in the first $(0$ to $<3.3)$ and second $(3.3-6.6$ $\mathrm{ng} / \mathrm{ml}$ ) quartiles had significantly increased ferritin levels, but not in the third and fourth quartiles. Their increased ferritin levels were much less than those observed in our study. HF is related to the inflammatory state, but the cause or consequence or both of HF is still being debated [13] and warrants further investigation [1].

The total amount of iron per day in our study was $65.96 \mathrm{mg}$, which is lower 
than in other studies. Despite this, the uptake was already significant after 6 weeks. This has never been shown before in patients with HF and it does not seem to differ if patients have HFrEF or HFpEF. This finding is in line with the results reported by Hallberg and Brise, who showed that iron succinate had the best absorption of all tested iron complexes [8] in healthy subjects. They also showed that succinate itself increased iron uptake when given intravenously, and they speculated that succinate influenced membrane transportation [8]. Succinate not only plays a role in energy metabolism, but is also an important metabolite at the cross-roads of several metabolic pathways, including endocrine and paracrine modulation, inflammation, and heme metabolism [14]. The above factors were important reasons to use iron succinate in our pilot study.

The adverse effects reported in our study were few, but well-known and expected. The overall compliance was good, and our findings may be due to the rather low iron dose.

We want to emphasize that we did not aim to study outcome in the present study, rather challenge the statement that oral iron therapy cannot be adequately taken up in patients with HF. For the future, we propose that iron succinate at doses as used in the present pilot study should be compared to iron sulfate in patients with HF, both HFrEF and HFpEF, to test whether any significant differences exist between the iron preparations.

Iron succinate should also be tested against intravenous iron ferric carboxymaltose in regards to clinical symptoms, quality of life, and hospitalization. We think it is important to determine if any oral iron therapy can be used in patients with HF given the very large number of patients with ID, one of the most prevalent co-morbid conditions in HF. An estimated $50 \%$ of patients with HF seem to have ID [15]. From a practical point of view and costs ( $~ 5 \%-10 \%$ of intravenous treatment, personnel communication), an oral therapy would be most valuable. In patients who do not respond, or due to side effects or a need for rapid improvement, intravenous iron therapy is already available and preferable.

This is a small pilot single-center study and selection bias cannot be excluded. Lack of a placebo control is an important limitation, but placebo did not significantly increase uptake in other studies. For example, Lewis et al. reported that the ferritin level and TSAT increased only $1 \mathrm{ng} / \mathrm{ml}$ and $0 \%$, respectively [5]. The only way in our study to increase iron levels should be dietary changes, but our patients were told not to change their diet habits throughout the study. An increase of ferritin levels of at least $50 \%$ is far from what could be expected with placebo treatment [5].

\section{Conclusion}

Oral iron succinate significantly increased iron uptake, almost doubling the ferritin levels and increasing TSAT, in patients with HF. Patients with HFrEF or HFpEF seemed to respond similarly. The role of succinate in iron uptake merits further investigation. 


\section{Conflicts of Interest}

The authors declare no conflicts of interest regarding the publication of this paper.

\section{References}

[1] Ponikowski, P., van Veldhuisen, D.J., Comin-Colet, J., Ertl, G., Komajda, M., Mareev, V., McDonagh, T., Parkhomenko, A., Tavazzi, L., Levesque, V., Mori, C., Roubert, B., Filippatos, G., Ruschitzka, F. and Anker, S.D. (2015) Beneficial Effects of Long-Term Intravenous Iron Therapy with Ferric Carboxymaltose in Patients with Symptomatic Heart Failure and Iron Deficiency. European Heart Journal, 36, 657-668. https://doi.org/10.1093/eurheartj/ehu385

[2] Anker, S.D., Kirwan, B.A., van Veldhuisen, D.J., Filippatos, G., Comin-Colet, J., Ruschitzka, F., Luscher, T.F., Arutyunov, G.P., Motro, M., Mori, C., Roubert, B., Pocock, S.J. and Ponikowski, P. (2018) Effects of Ferric Carboxymaltose on Hospitalisations and Mortality Rates in Iron-Deficient Heart Failure Patients: An Individual Patient Data Meta-Analysis. European Journal of Heart Failure, 20, 125-133. https://doi.org/10.1002/ejhf.823

[3] McDonagh, T. and Macdougall, I.C. (2015) Iron Therapy for the Treatment of Iron Deficiency in Chronic Heart Failure: Intravenous or Oral? European Journal of Heart Failure, 17, 248-262. https://doi.org/10.1002/ejhf.236

[4] Markousis-Mavrogenis, G., Tromp, J., Ouwerkerk, W., Devalaraja, M., Anker, S.D., Cleland, J.G., Dickstein, K., Filippatos, G.S., van der Harst, P., Lang, C.C., Metra, M., Ng, L.L., Ponikowski, P., Samani, N.J., Zannad, F., Zwinderman, A.H., Hillege, H.L., van Veldhuisen, D.J., Kakkar, R., Voors, A.A. and van der Meer, P. (2019) The Clinical Significance of Interleukin-6 in Heart Failure: Results from the BIOSTAT-CHF Study. European Journal of Heart Failure, 21, 965-973.

https://doi.org/10.1002/ejhf.1482

[5] Lewis, G.D., Malhotra, R., Hernandez, A.F., McNulty, S.E., Smith, A., Felker, G.M., Tang, W.H.W., LaRue, S.J., Redfield, M.M., Semigran, M.J., Givertz, M.M., Van Buren, P., Whellan, D., Anstrom, K.J., Shah, M.R., Desvigne-Nickens, P., Butler, J., Braunwald, E. and Network NHFCR (2017) Effect of Oral Iron Repletion on Exercise Capacity in Patients with Heart Failure with Reduced Ejection Fraction and Iron Deficiency: The IRONOUT HF Randomized Clinical Trial. JAMA, 317, 1958-1966. https://doi.org/10.1001/jama.2017.5427

[6] Ponikowski, P., Voors, A.A., Anker, S.D., Bueno, H., Cleland, J.G.F., Coats, A.J.S., Falk, V., Gonzalez-Juanatey, J.R., Harjola, V.P., Jankowska, E.A., Jessup, M., Linde, C., Nihoyannopoulos, P., Parissis, J.T., Pieske, B., Riley, J.P., Rosano, G.M.C., Ruilope, L.M., Ruschitzka, F., Rutten, F.H., van der Meer, P. and Group ESCSD (2016) 2016 ESC Guidelines for the Diagnosis and Treatment of Acute and Chronic Heart Failure: The Task Force for the Diagnosis and Treatment of Acute and Chronic Heart Failure of the European Society of Cardiology (ESC) Developed with the Special Contribution of the Heart Failure Association (HFA) of the ESC. European Heart Journal, 37, 2129-2200. https://doi.org/10.1093/eurheartj/ehw128

[7] Brise, H. and Hallberg, L. (1962) Absorbability of Different Iron Compounds. Acta Medica Scandinavica Supplementum, 171, 23-37. https://doi.org/10.1111/j.0954-6820.1962.tb18680.x

[8] Brise, H. and Hallberg, L. (1962) Effect of Succinic Acid on Iron Absorption. Acta Medica Scandinavica Supplementum, 171, 59-73.

https://doi.org/10.1111/j.0954-6820.1962.tb18684.x 
[9] Grimolizzi, F. and Arranz, L. (2018) Multiple Faces of Succinate beyond Metabolism in Blood. Haematologica, 103, 1586-1592. https://doi.org/10.3324/haematol.2018.196097

[10] Li, H., Rose, M.J., Tran, L., Zhang, J., Miranda, L.P., James, C.A. and Sasu, B.J. (2009) Development of a Method for the Sensitive and Quantitative Determination of Hepcidin in Human Serum Using LC-MS/MS. Journal of Pharmacological and Toxicological Methods, 59, 171-180. https://doi.org/10.1016/j.vascn.2009.02.004

[11] Beck-da-Silva, L., Piardi, D., Soder, S., Rohde, L.E., Pereira-Barretto, A.C., de Albuquerque, D., Bocchi, E., Vilas-Boas, F., Moura, L.Z., Montera, M.W., Rassi, S. and Clausell, N. (2013) IRON-HF Study: A Randomized Trial to Assess the Effects of Iron in Heart Failure Patients with Anemia. International Journal of Cardiology, 168, 3439-3442. https://doi.org/10.1016/j.ijcard.2013.04.181

[12] Gaillard, C.A., Bock, A.H., Carrera, F., Eckardt, K.U., Van Wyck, D.B., Bansal, S.S., Cronin, M., Meier, Y., Larroque, S., Roger, S.D. and Macdougall, I.C. (2016) Hepcidin Response to Iron Therapy in Patients with Non-Dialysis Dependent CKD: An Analysis of the FIND-CKD Trial. PLoS ONE, 11, e0157063. https://doi.org/10.1371/journal.pone.0157063

[13] Van Linthout, S. and Tschope, C. (2017) Inflammation-Cause or Consequence of Heart Failure or Both? Current Heart Failure Reports, 14, 251-265. https://doi.org/10.1007/s11897-017-0337-9

[14] Tretter, L., Patocs, A. and Chinopoulos, C. (2016) Succinate, an Intermediate in Metabolism, Signal Transduction, ROS, Hypoxia, and Tumorigenesis. Biochimica et Biophysica Acta, 1857, 1086-1101. https://doi.org/10.1016/j.bbabio.2016.03.012

[15] Klip, I.T., Comin-Colet, J., Voors, A.A., Ponikowski, P., Enjuanes, C., Banasiak, W., Lok, D.J., Rosentryt, P., Torrens, A., Polonski, L., van Veldhuisen, D.J., van der Meer, P. and Jankowska, E.A. (2013) Iron Deficiency in Chronic Heart Failure: An International Pooled Analysis. American Heart Journal, 165, 575-582. https://doi.org/10.1016/j.ahj.2013.01.017 